\title{
1 The evolution of red blood cell shape in a continental radiation of fishes
}

3 Brenda Oliveira Martins ${ }^{*}$, Lilian Franco-Belussi ${ }^{2 *}$, Mayara Schueroff Siqueira $^{2}$, Carlos

$6{ }^{1}$ Graduate Program in Animal Biology. Instituto de Biociências, Universidade Federal

7 de Mato Grosso do Sul, Campo Grande, Mato Grosso do Sul, 79002970, Brasil.

8 brendabernini@gmail.com

$9{ }^{2}$ Instituto de Biociências, Universidade Federal de Mato Grosso do Sul, Campo 10 Grande, Mato Grosso do Sul, 79002970, Brasil. lilian.belussi@gmail.com;

11 carlos.fernandes@ufms.br; mayara_schueroff@hotmail.com

$12{ }^{3}$ Gothenburg Global Biodiversity Centre, Göteborg, Box 100, S 405 30, Sweden.

13 "These authors contributed equally to this work

15 Short running title: RBC shape in fishes

17 Author contributions: BOM collected cell morphometric data assisted by MSS, LFB.

18 CEF and DBP conceived the study. DBP analysed and curated the data. DBP, LFB, and

19 BOM prepared the first draft of the manuscript to which all authors contributed

20 afterwards.

22 Corresponding author:

23 Diogo B. Provete

24 e-mail: diogo.provete@ufms.br

25 Instituto de Biociências - INBIO

26 Universidade Federal de Mato Grosso do Sul - UFMS, Av. Costa e Silva, s/n, Bairro

27 Universitário, Campo Grande, 79002-970, Mato Grosso do Sul, Brasil 


\section{Abstract}

31 The size and shape of Red Blood Cells (RBC) can provide key information on life

32 history strategies in vertebrates. However, little is known about how RBC shape

33 evolved in response to environmental factors and the role of phylogenetic relationship.

34 Here, we analyzed RBC morphometrics in a continental radiation of fishes testing the

35 hypothesis that phylogenetic relationship determines species occupation of

36 morphospace. We collected blood samples of five specimens of 15 freshwater fish

37 species from six orders and used basic stereological methods to measure cell and

38 nucleus area, perimeter, and diameter, cell and nucleus volume, nucleus:cytoplasm

39 ratio, and shape factor of 50 cells per specimen. Then, we conducted a phylogenetic

40 Principal Components Analysis using a dated phylogeny and built a phylomorphospace.

41 To test if the phylogenetic relationship predicted the phenotypic similarity of species,

42 we calculated multivariate phylogenetic signal. We also estimated the evolution rate of

43 RBC shape for each node and tip using ridge regression. Finally, we tested if the

44 position in the water column influenced RBC shape using a phylogenetic GLS. RBC

45 shape seems to have evolved in a non-stationary way because the distribution pattern of

46 species in the phylomorphospace is independent of the phylogeny. Accordingly, the rate

47 of evolution for shape was highly heterogeneous, with an increase in the genus

48 Pygocentrus. Water column position does not influence RBC shape. In conclusion,

49 RBC shape seem to have evolved in response to multiple selective pressures

50 independent of life history characters.

51 Key-words: phylomorphospace, erythrocytes, stereology, phylogenetic signal, rate of

52 evolution.

53

54

55

\section{Introduction}


56 The shape, size, and number of Red Blood Cells (RBC) vary greatly in

57 vertebrates (Wintrobe 1934). Although the reasons for this variation are not well

58 understood, phylogenetic relationships can at least partially explain it, because early

59 diverging vertebrate lineages have larger but fewer RBC (Anderson et al., 2018). Early

60 diverging vertebrates also have round and flattened RBCs with a central nucleus, while

61 derived lineages have ellipsoid cells (Nikinmaa 1990; Glomski et al. 1992).

62 Both abiotic factors and life history traits can also affect the composition and 63 morphology of fish RBC (Dal'Bó et al., 2015; Kumar, 2016; Yakhnenko et al., 2016).

64 For example, RBC size is inversely related to swimming ability in teleosts (Lay and

65 Baldwin 1999; Witeska 2013), while size of RBCs is highest at intermediate altitude in

66 one lizard species (González-Morales et al. 2017). In addition, cell size has an inverse

67 relation with metabolic rate, suggesting lowest metabolic cost to maintain active

68 membrane transport in large cells (Szarki, 1970; Graham et al. 1985, Maciak et al,

69 2011). Deep-sea fish species have large RBC, compatible with their low metabolism

70 (Graham et al. 1985). Then, RBC size in teleost has an adaptative value and changes in

71 response to external factors (Lay and Baldwin 1999). RBC volume is another

72 morphological variable correlated with physiological state in fish. For example, cell

73 volume is negative correlated with hemoglobin concentration, while nuclear volume

74 explains only 17\% of cell volume (Lay and Baldwin 1999).

75 The nucleus of RBC is strongly related to species adaptation to environmental

76 conditions. Nucleus size is positively correlated with genome size in vertebrates

77 (Gregory 2001). Accordingly, the amount of DNA regulates the size of cells as well as

78 the nucleus. Larger nuclei have more room for protein synthesis that are required in the

79 metabolism of larger cells (Gregory 2001). Variations in RBC DNA content are also

80 directly related to hematopoiesis, which in turn influence species adaptation. Genome 
81 size seems also related to life-history traits. For example, genome size is positively

82 related with the size and diameter of eggs, and negatively related with growth rate and

83 standard size in fish (Hardie and Hebert, 2004; Smith and Gregory 2009).

84 The shape of RBC also changes according to cell maturation stage. While

85 immature RBC (erythroblasts) are usually rounded, older cells are fusiform (Fijan 2002,

86 Passantino et al. 2004). However, the degree of ellipticalness seems to vary among

87 species (Dal'Bó et al., 2015). However, little is known about the physiological

88 implications of the variation in the fusiform shape for species. Similarly, little is known

89 about the factors involved in the variation of RBC volume and its implications (Lay and

90 Bladwin 1999) but increasing temperature and body mass increase RBC volume in

91 tetrapods (Gillooly and Zenil-Ferguson 2014). Also, RBC volume is related to

92 polyploidy in fish (Gregory 2001). However, little is known about the relative role of

93 phylogenetic relationships and abiotic factors in determining RBC shape.

94 No study has yet analysed how RBC shape have evolved in fish using modern

95 phylogenetic comparative methods. As shape is multivariate, here we used a set of first-

96 and second-order stereological variables to describe the shape of RBC. Also, we tested

97 if the position in the water column, as a proxy for partial oxygen pressure, species are

98 usually found affect RBC shape. Our hypothesis is that neustonic species (that live

99 closer to the surface) would have smaller RBC due to higher surface area to volume

100 ratio. We also built the phylomorphospace of RBC shape and tested for phylogenetic

101 signal. We expect species with similar habitat use would be close together in the

102 morphospace. This is the first study combining common stereological analysis and

103 phylogenetic comparative method to understand how fish RBC evolved. Our results can

104 help understand how fish species evolved to occupy habitats with different oxygen 
105 concentration or toxicity, besides understanding how metabolic rate vary among

106 species.

107

108

Methods

109

Specimen sampling

We analyzed five individuals of 15 species belonging to 15 genera and 2

111 superorders: Pygocentrus nattereri, Gymnotus inaequilabiatus, Astyanax lineatus,

112 Bujurquina vittata, Cichlasoma dimerus, Metynnis maculatus, Prochilodus lineatus,

113 Piaractus mesopotamicus, Rhamdia quelen, Poecilia reticulata, Hypostomus

114 boulengeri, Pseudoplatystoma corruscans, Synbranchus marmoratus, Brycon hilarii,

115 and Hyphessobrycon anisitsi (Figure 1). We obtained adult fish specimens from both

116 sexes between 2017 and 2018 from a number of sources: Pygocentrus nattereri was

117 fished in a river the Pantanal field station (Corumbá, Mato Grosso do Sul, central

118 Brazil); Gymnotus inaequilabiatus, Hyphessobrycon anisitsi, and Synbranchus

119 marmoratus were bought from a fish store; Astyanax lineatus and Bujurquina vittata

120 were collected in an urban lake in Campo Grande; Cichlasoma dimerus, Metynnis

121 maculatus, Prochilodus lineatus, Piaractus mesopotamicus were donated by the city

122 aquarium; Pseudoplatystoma corruscans, Brycon hilarii, and Hypostomus boulengeri

123 were obtained from the university Aquaculture Research ponds; Poecilia reticulata was

124 collected in an urban stream in Campo Grande. These species were selected because

125 they are common in our region, use distinct habitats, and belong to distant lineages.

126 Fish were anaesthetized with eugenol solution $\left(50 \mathrm{mg} \mathrm{L}^{-1}\right)$ before blood

127 sampling. Blood was collected by caudal vein or cardiac puncture with syringe with $3 \%$

128 EDTA or by decapitation in small species (Ranzani-Paiva et al., 2013). Blood smears

129 were stained with May-Grunwald-Giemsa-Wright for erythrocyte morphometry 
130 (Tavares-Dias and Moraes 2002). Detailed protocol is available at Rodrigues et al.

131 (2020; see also Figure S1).

132

\section{Red blood cell stereology}

134 Design-based stereological methods have been used since the 1970s to quantify

135 shape and size changes in cells and histological structures (West 2012). This approach

136 uses linear (first-order) measurements to derive tridimensional (second-order) variables

137 that describe the shape of cells in tissues. To take measurements, we used a digital

138 camera (Nikon D3500) coupled in a light microscopy (Zeiss Primo Star ${ }^{\circledR}$ ). Digital

139 images in 1000x magnification were used to measure the following first-order variables:

140 area $\left(\mu \mathrm{m}^{2}\right)$, perimeter, and largest and smallest diameter $(\mu \mathrm{m})$ of cells and area,

141 perimeter, and diameter of the nucleus in 50 randomly selected cells per animal (see

142 Supporting material), i.e., 250 cells per species (50 cells * 5 animals). From these linear

143 measurements, we calculated the following second-order stereological measurements:

144 To calculate length, we took the mean of the largest and smallest diameters. Then, to

145 calculate radius, we simply divided length by 2 . Cell volume $\left(\mu \mathrm{m}^{3}\right)$ was calculated using

146 the formula:

$$
\frac{4}{3 \pi} r^{3}
$$

147 , where $r$ is radius (see West 2012); nucleus volume $\left(\mu \mathrm{m}^{3}\right)$ was calculated by the

148 formula:

$$
\frac{C V * N: C}{100}
$$

149 , where $\mathrm{CV}$ is cell volume and $\mathrm{N}: \mathrm{C}$ is nucleus:cytoplasm ratio. Cell and nucleus

150 circularity (or shape factor) were estimated by the formula:

$$
\frac{4 \pi A}{P^{2}}
$$


151 , where $A$ is cell area and $P$ cell perimeter (Russ and Dehoff 2000). Circularity ranges

152 from 0 (elliptical shape) to 1 (circular shape). All measurements were made in Motic

153 Images Plus 2.0 in a double-blind fashion (see Fig. S2).

154 We calculated the mean of each variable for each species (Table S1), which were

155 used as phenotypic traits in further analysis. To visualize the correlation pattern between

156 variables, we built a correlogram considering the average of each variable (Fig. S3)

157 using the corrplot package (Wei and Simko, 2017) of R software.

159 Phylogenetic comparative methods

160 To carry out the comparative analyzes, we first obtained a phylogeny with

161 branch length in millions of years representing the relationship of the 15 species for

162 which we have phenotypic data (Figure 1). This phylogeny was obtained from a fully-

163 sampled topology for Actinopterygii recently published (Rabosky et al., 2018). Analysis

164 was done in the fishtree package (Chang et al., 2019).

165 Then, we performed a phylogenetic Principal Components Analysis (pPCA) on

166 the matrix of phenotypic data with the eight variables considering the phylogeny

167 (Revell, 2009). The pPCA was performed using the correlation matrix of variables and

168 assuming that traits have evolved according to a Brownian Motion (BM) model of

169 evolution. The pPCA uses a $\mathbf{C}$ matrix that describes the variance-covariance structure

170 between species given by the phylogeny and the evolutionary model (BM in this case)

171 to obtain the Principal Components and species scores (see also Adams \& Collyer

172 2019). Analysis was performed using the phyl.pca function of the phytools package

173 (Revell, 2012).

174 From this exploratory analysis, we built a morphospace for all 15 species

175 showing their similarity in relation to RBC shape. The analysis estimates the node 
176 values using a Maximum Likelihood method. Finally, we built the phylomorphospace

177 by projecting the phylogeny onto the morphospace built with the first two Principal

178 Components, which allows us to explore how lineages occupied the morphospace as

179 their traits evolved (Adams and Coller 2018, 2019). Analysis was performed using the

180 phylomorphospace function of the phytools package.

181 Finally, we calculated a phylogenetic signal measure for multivariate data called

$182 \mathrm{~K}_{\text {mult }}$ (Adams, 2014) using all pPCs to avoid missing variation (Uyeda et al., 2015). This

183 method allows calculating a statistic that measures how similar closely related species

184 are in relation to their phenotypic measurements. Blomberg's K (Blomberg et al., 2003)

185 is a measure widely used to measure phylogenetic signal for univariate data and ranges

186 from $0<\mathrm{K}>1$. High phylogenetic signal $(\mathrm{K}>1)$ indicates that closely related species

187 tend to be similar in relation to phenotypic attributes (Münkemüller et al., 2012). This

188 method has been adapted for multivariate data recently (Adams, 2014), along with a

189 Monte Carlo randomization procedure that allows testing the significance of the

190 statistic. Analysis was performed with the physignal function of the geomorph

191 package (Adams et al. 2020).

192 To test the effect of the position in the water column species usually occupy on

193 RBC shape, we gathered data on depth range from FishBase (Froese and Pauly 2019)

194 and treated it as a categorical variable, henceforth referred to as habitat. Thus, species

195 were classified as demersal $(n=4)$, pelagic $(n=2)$, and benthopelagic $(n=9)$. Afterwards,

196 we compared the fit of evolutionary models to the eight pPCs. Specifically, we fit

197 Pagel's lambda transformation, Brownian Motion, and Ornstein-Uhlenbeck models to

198 data using the leave one out cross-validation of the penalized log-likelihood, as

199 implemented in the mvgls function (Clavel and Morlon 2020) of the package

200 mvMORPH (Clavel et al. 2015) including habitat as a predictor variable. Then, we 
201 compared the model fit using the Extended Information Criterion (EIC), which showed

202 that the best model was Brownian Motion (BM; Table S2). We built a phylogenetic

203 Generalized Least Squares (Clavel and Morlon 2020) using BM to test the effect of

204 habitat on RBC shape, with the Pillai's trace as test statistic and Type I Sum of Squares.

205 Finally, to test the hypothesis if a change in evolution rate is the mechanism

206 allowing species to occupy different positions in the morphospace, we estimated tip-

207 level evolution rate of RBC shape using phylogenetic ridge regression, as implemented

208 in the RRphylo package (Castiglione et al. 2018). For this analysis we used all eight

209 pPCs. This analysis also automatically finds nodes in which there was a rate shift. The

$210 \mathrm{R}$ markdown dynamic document describing how analyses were conducted and

211 associated data are available at FigShare (Martins et al. 2020).

The shape of Red Blood Cells (RBC) of fish species studied varied from oval to 216 ellipsoid and a rounded nucleus (Figure 2). Cell size varied by an order of magnitude, 217 from the largest cell of Synbranchus to the smallest in Poecilia. The same phenomenon 218 happened to cell volume (Fig. 3). Nucleus area varied from $7.45 \mu \mathrm{m}^{2}( \pm 1.2 \mathrm{SD})$ in 219 Poecilia to $27.30 \mu \mathrm{m}^{2}( \pm 4.9 \mathrm{SD})$ in Synbranchus. Additionally, the range of 220 intraspecific variation in some measurements varied among species. For example, cell 221 volume had the highest standard deviation, while shape factor, nucleus area and cell 222 perimeter seem to vary less within species (Table S1).

223 The $\mathrm{pPC} 1$ retained $66.1 \%$, while $\mathrm{pPC} 2$ retained $21.4 \%$ of the variation in $\mathrm{RBC}$ 224 shape. Cell and nucleus area, cell and nucleus perimeter, cell and nucleus volume were 225 highly negatively correlated (> 0.84) with pPC1, whereas the nucleus:cytoplasm ratio 226 was highly (-0.87) correlated with pPC2 (Table S2). Species positively correlated with 
227 pPC1 had higher shape factor, whereas those negatively correlated had higher cell and

228 nucleus area, nuclear and cell perimeter, cell and nucleus volume, and

229 nucleus:cytoplasm ratio (Table S2). Species positively correlated with pPC2 had higher

230 cell perimeter, cell and nuclear area, whereas those negatively correlated had higher

231 nucleus:cytoplasm ratio, nucleus and cell volume, and nucleus area (Fig. S4).

232 The distribution of species in the phylomorphospace suggest that closely related

233 species do not have RBC with similar shape (Fig. 4). Accordingly, RBC shape does not

234 exhibit phylogenetic signal $\left(\mathrm{K}_{\text {mult }}=0.6317\right.$; Effect size $\left.=0.7863 ; P=0.159\right)$. The low

235 effect size suggest phylogenetic signal is not concentrated in a few dimensions. To test

236 if only a subset of dimensions displays phylogenetic signal (see Adams \& Collyer

237 2019), we re-run the analysis with only the first two pPCs. Results did not change (see

238 Martins et al. 2020). However, the morphospace suggest a non-stationary pattern in the

239 evolution of RBC shape, since most closely related species occupy distant positions, but

240 the position of siluriformes (Pseudoplatistoma, Rhamdia, and Hypostomus) mirror their

241 phylogenetic relationship in the upper part of the morphospace. Interestingly, the

242 Acanthopterygii included in our sampling: Synbranchus, Poecilia, Bujurquina, and

243 Cichlosoma had the most divergent pattern, occupying extreme positions in the

244 phylomorphospace, whereas species of Ostariophysi were more packed together. There

245 is a high divergence in $\mathrm{RBC}$ shape within Characiformes. Therefore, there seems to be a

246 variation in the pattern of RBC shape evolution at the level of higher order groups

247 (Superorders), instead of a homogeneous pattern throughout the whole phylogeny.

248 Habitat did not influence RBC shape (Pillai's trace $=0.6936 ; P=0.461$ ). The

249 results did not change if we use a multivariate PGLS (Table S4). We also found that

250 evolution rate of RBC shape was very heterogeneous at the tip level (Fig. 5). The

251 species with the highest rate was Pygocentrus (0.741) and the lowest was Bujurquina 
252 (0.087). There was a tendency to increase the rate in the common ancestor of

253 Pygocentrus, Metynnis, and Piaractus in relation to the background rate, but the rate

254 shift was not significant (rate difference $=0.392 ; P>0.005$ ). Interestingly, the species

255 occupying the most extreme positions in the phylomorphospace, such as Synbranchus

256 (0.514), Astyanax (0.555), and Gymnotus (0.17) were not the ones with the highest rates

257 of evolution. Therefore, our results suggest that an increase in the rate of evolution does

258 not necessarily produces morphological specialization and rate disparity is not the main

259 pattern involved in the formation of RBC morphospace.

260

261

262

263

264

265

266

267

268

269

270

271

272

274

275

276

277

\section{Discussion}

We found that the shape of Red Blood Cells (RBC) varies greatly among and within freshwater fish species. Contrarily to our initial hypothesis, we did not find a correlation between water column position (proxy for oxygen availability) and RBC shape. Accordingly, we did not find phylogenetic signal in RBC shape, suggesting closely related species do not have similar RBC shape. There does not seem to be a single mechanism driving the evolution of RBC shape, since the phylomorphospace shows a non-stationary pattern in the species similarity.

Small RBC allow species to occupy environments with low dissolved oxygen, since they usually have a higher number of cells, making them more efficient in oxygen uptake and transport (Silkin et al., 2019; Hawkey et al., 1991). Species with RBCs that have large surface area and volume have low metabolic rate, since the cell surface:volume ratio decreases the metabolic cost of gas exchange between membranes (Jones 1979, Lay and Baldwin, 1999). Synbranchus had higher perimeter (i.e., higher surface) and higher area. Thus, our results suggest that this species could have a lower metabolic rate than Poecilia, because the later has a smaller cell area and perimeter. 
Most species occupied the center of the morphospace, independent of the

279 phylogenetic relationship. This clustering of distantly related species suggests that

280 convergence, apparently not caused by water column position, could be a mechanism

281 influencing RBS shape evolution (Adams and Collyer 2019). Clustering in

282 morphospace can also suggest a developmental constraint (Jablonski 2020) that impedes

283 species to change their traits after speciation (Pie and Weitz 2005). Also, a few species

284 were in extreme positions. Even though water column position did not explain RBC

285 shape, the extreme position of Synbranchus, Astyanax, and Poecilia in the morphospace

286 could be explained by differences in their life history strategies. For example,

287 Synbranchus displays facultative pulmonary respiration, usually occurs in habitats with

288 hypoxia, and burry itself during the dry season (Froese and Pauly, 2019). Astyanax also

289 occurs in habitats with hypoxia (Froese and Pauly, 2019). The pattern we found points

290 to clade disparity differences (Adams \& Collyer 2019), with Acanthopterygii having

291 higher dispersion in the morphospace than Ostariophysi (Fig. S6). These two orders

292 have different ecological and biogeographic characteristics. Species from Ostariophysi

293 are essentially from freshwater, while Acanthopterygii has marine origins, with

294 secondary freshwater introgressions. One interesting pattern that emerges from the

295 morphospace is that apparently there is no constrains in the morphospace, because

296 almost all regions have one species. Siluriformes seem to have similar RBC shape,

297 given their position in the morphospace, and similar evolution rate. This lineage

298 occupies the upper position of the morphospace, along with Piaractus and

299 Hyphessobrycon, and had high cell area and perimeter, and low nucleus:cytoplasm ratio,

300 nucleus volume, and circularity. Exception for Rhamdia and Hyphessobrycon, all those

301 species are demersal (benthic). Together these results suggest that the evolution of 
302 RBC shape was driven by fluctuating selection, although this result should be taken

303 with care, since our sample size is not large.

304 We found a high heterogeneity in evolution rate of RBC shape. This high rate

305 heterogeneity helps explain why we did not detect a significant phylogenetic signal, i.e.,

306 the change in shape seems to vary strongly at the tip level, instead of large clades. This

307 is consistent with the model selection procedure that pointed Brownian Motion (BM) as

308 best fit model to the data. BM can be caused by genetic drift, when selection randomly

309 varies in direction through time, or when selection is weak compared to the time scale

310 analysed (Felsenstein 1988). The large intraspecific variation in most variables (Table

311 S1) may suggest that variables describing RBC shape are under weak selection pressure

312 (Nikinmaa 2019). Interestingly, the position extreme positions of Synbranchus (0.514),

313 Astyanax (0.555), and Pygocentrus (0.741) could be explained at least partially by an

314 increase in evolution rate, which allowed them to diverge from their sister species,

315 remaining Acanthopterygii, Hyphessobrycon, and Metynnis respectively. However,

316 given our sample size we cannot rule out that RBC shape of these species are evolving

317 under directional selection. Conversely, if lineages followed a random walk (akin of

318 BM) one would expect species to diverge and occupy positions in the morphospace

319 irrespective of their habitat or phylogenetic position (Pie and Weitz 2005).

320 Water column position did not explain RBC shape in this freshwater fish species

321 radiation. This result suggest that RBC shape may not be entirely determined by partial

322 pressure of dissolved oxygen in water. It also suggests that water column position does

323 not represent a main constraint in the evolution of these traits. A recent study (Minias

324 2020) also found that life history traits did not explain hemoglobin and hematocrit

325 evolution in birds. Differently from mammals, fish RBC are nucleated cells and have

326 many cytoplasmic organelles, such as mitochondria, Golgi complex, centrioles, 
327 ribosomes, microtubules, rough and smooth endoplasmic reticulum (Sekhon and Beams

328 1969), as well as a range of cytoplasmatic enzymes involved in the anaerobic glycolysis

329 (Sephton et al. 1991). These organelles and enzymes allow the functions of RBC to be

330 finely regulated and somewhat independent from other components of the

331 hematopoietic system, enabling fish to occupy a wide range of environments (Nikinmaa

332 et al. 2019). Therefore, species may respond rapidly to changes in oxygen availability

333 (Witeska, 2013), which indicates that it is not constrained by inheritance (Arnold 1992)

334 or phylogenetic inertia, at least to a certain degree.

335 In conclusion, our study provides a novel macroevolutionary perspective on fish

336 RBC shape by combining commonly used design-based stereological techniques with

337 modern phylogenetic comparative methods. We found that the RBC of the two main

338 orders of Neotropical freshwater fish vary greatly in shape, but that their water column

339 alone does not explain it. Future studies should use quantitative genetic approaches to

340 explore the underlying genetic basis for RBC morphology, possibly using geometric

341 morphometrics and experimental approaches motivated by ecological data.

342

343

344

345

346

347

348

349

350

351

352

353

354

\section{Acknowledgements}

This study was supported by Universidade Federal de Mato Grosso do Sul -

UFMS/MEC - Brasil. This study was funded in part by the Coordenação de

Aperfeiçoamento de Pessoal de Nível Superior - Brasil (CAPES) - Finance Code 001.

F. R. Carvalho and K. Tondato provided insightful comments as members of BOM

bachelor's honors thesis examining committee.

\section{Literature cited}

Adams, D. C. 2014. A generalized K statistic for estimating phylogenetic signal from shape and other high-dimensional multivariate data. Syst Biol 63:685-697. 
355 Adams, D. C., M. L. Collyer, and A. Kaliontzopoulou. 2020. Geomorph: Software for

356 geometric morphometric analyses. R package version 3.2.1. https://cran.r-

$357 \quad$ project.org/package=geomorph.

358 Adams, D. C. and M. L. Collyer. 2018. Multivariate Phylogenetic Comparative Methods:

359 Evaluations, Comparisons, and Recommendations. Syst Biol 67:14-31.

360 Adams, D. C. and M. L. Collyer. 2019. Phylogenetic Comparative Methods and the Evolution

361 of Multivariate Phenotypes. Annual Review of Ecology, Evolution, and Systematics 50:

$362 \quad 405-425$.

363 Anderson, H. L., I. E. Brodsky, N. S. Mangalmurti. 2018. The Evolving Erythrocyte: Red

364 Blood Cells as Modulators of Innate Immunity. Journal of Immunology 201: 1343-

$365 \quad 1351$.

366 Arnold, S. J. 1992. Constraints on phenotypic evolution. Am Nat 140 Suppl 1:S85-107.

367 Blomberg, S. P., T. Garland, Jr., and A. R. Ives. 2003. Testing for phylogenetic signal in

368 comparative data: behavioral traits are more labile. Evolution 57:717-745.

369 Castiglione, S., G. Tesone, M. Piccolo, M. Melchionna, A. Mondanaro, C. Serio, M. Di

370 Febbraro, P. Raia, and N. Cooper. 2018. A new method for testing evolutionary rate

$371 \quad$ variation and shifts in phenotypic evolution. Methods in Ecology and Evolution 9:974-

372983.

373 Chang, J., D. L. Rabosky, S. A. Smith, M. E. Alfaro, and D. Silvestro. 2019. An R package and

374 online resource for macroevolutionary studies using the ray $\square$ finned fish tree of life.

$375 \quad$ Methods in Ecology and Evolution 10:1118-1124.

376 Clavel, J., G. Escarguel, G. Merceron, and T. Poisot. 2015. mvmorph: an R package for fitting

377 multivariate evolutionary models to morphometric data. Methods in Ecology and

$378 \quad$ Evolution 6:1311-1319. 
379 Clavel, J. and H. Morlon. 2020. Reliable Phylogenetic Regressions for Multivariate

380 Comparative Data: Illustration with the MANOVA and Application to the Effect of Diet

381 on Mandible Morphology in Phyllostomid Bats. Syst Biol.

Dal'Bó, G.A., Sampaio, F.G., Losekann, M.E., Queiroz, J.F.D., Luiz, A.J. B., Wolf, V.H.G., Ichthyology 13:439-446.

Felsenstein, J. 1988. Phylogenies and Quantitative Characters. Annual Review of Ecology and Systematics 19:445-471.

Fijan, N. 2002. Morphogenesis of blood cell lineages in channel catfish. Journal of fish biology 60: 999-1014.

Froese, R. and D. Pauly. 2019. FishBase. World Wide Web electronic publication.

Gillooly, J. F. and R. Zenil-Ferguson. 2014. Vertebrate blood cell volume increases with temperature: implications for aerobic activity. PeerJ 2:e346.

Glomski, C. A., Tamburlin, J., Chainani, M. 1992. The phylogenetic odyssey of the 501-28. 645.

400 Graham, M.S., Haedrich, R.L. and Fletcher, G.L. 1985. Hematology of three deep-sea fishes: A 
403 Gregory, T. R. 2001. The bigger the C-value, the larger the cell: genome size and red blood cell 404 size in vertebrates. Blood Cells, Molecules, and Diseases 27:830-843.

405 Hawkey, C., P. Bennett, S. Gascoyne, M. Hart, and J. Kirkwood. 1991. Erythrocyte size, 406 number and haemoglobin content in vertebrates. British journal of haematology 77:392$407 \quad 397$.

408 Hardie, D.C. and Hebert, P.D. 2003. The nucleotypic effects of cellular DNA content in 409 cartilaginous and ray-finned fishes. Genome 46:683-706.

410 Jablonski, D. 2020. Developmental bias, macroevolution, and the fossil record. Evolution \& 411 development 22:103-125.

412 Jones, D. 1979. The important of surface area/volume ratio to the rate of oxygen uptake by red $413 \quad$ cells. The Journal of general physiology 74:643-646.

414 Lay, P. A. and J. Baldwin. 1999. What determines the size of teleost erythrocytes? Correlations 415 with oxygen transport and nuclear volume. Fish Physiology and Biochemistry 20:31-35.

416 Kumar, M. V. 2016. Morphometric studies of blood cells in Cyprinus carpio, Ctenopharyngodan idella and Hypophthalmichthys molitrix cultured fish in west Godavari region of Andhra Pradesh. International Journal of Fisheries and Aquatic Studies 4: 489-493.

Maciak, S., Janko, K., Kotusz, J., Choleva, L., Boroń, A., Juchno, D., Kujawa, R., Kozłowski, J. and Konarzewski, M. 2011. Standard Metabolic Rate (SMR) is inversely related to erythrocyte and genome size in allopolyploid fish of the

424 Martins, B. O.; Franco-Belussi, L.; Siqueira, M. S.; Fernandes, C. E. S.; Provete, D. (2020): The evolution of red blood cell shape in a continental radiation of 
427 Minias, P. 2020. Ecology and evolution of blood oxygen-carrying capacity in birds.

428 American Naturalist 195. DOI: https://doi.org/10.1086/707720.

429 Münkemüller, T., S. Lavergne, B. Bzeznik, S. Dray, T. Jombart, K. Schiffers, and W.

430 Thuiller. 2012. How to measure and test phylogenetic signal. Methods in

$431 \quad$ Ecology and Evolution 3:743-756.

432 Nikinmaa, M. 1990. Vertebrate Red Blood Cells. Springer-Verlag, Berlin.

433 Nikinmaa, M. 2019. Environmental regulation of the function of circulating erythrocytes via

$434 \quad$ changes in age distribution in teleost fish: Possible mechanisms and

$435 \quad$ significance. Marine genomics 49:100717.

436 Nikinmaa, M., Berenbrink, M., \& Brauner, C. J. 2019. Regulation of erythrocyte function:

437 multiple evolutionary solutions for respiratory gas transport and its regulation in fish.

$438 \quad$ Acta Physiologica 227:e13299.

439 Passantino, L., Altamura, M., Cianciotta, A., Jirillo, F., Ribaud, M.R., Jirillo, E., and

440 Passantino, G.F. 2004. Maturation of Fish Erythrocytes Coincides with Changes in their

441 Morphology, Enhanced Ability to Interact with Candida albicans and Release of

442 Cytokine $\square$ Like Factors Active Upon Autologous Macrophages. Immunopharmacology

$443 \quad$ and immunotoxicology 26:573-585.

444 Pie, M. R. and J. S. Weitz. 2005. A Null Model of Morphospace Occupation. American

$445 \quad$ Naturalist 166: E1-13.

446 Rabosky, D. L., J. Chang, P. O. Title, P. F. Cowman, L. Sallan, M. Friedman, K. Kaschner, C.

447 Garilao, T. J. Near, M. Coll, and M. E. Alfaro. 2018. An inverse latitudinal gradient in

$448 \quad$ speciation rate for marine fishes. Nature 559:392-395.

449 Ranzani-de-Paiva, M. J. T., de Pádua, S. B., Tavares-Dias, M., \& Egami, M. I. 2013. Métodos

450 para análise hematológica em peixes. Editora da Universidade Estadual de Maringá-

$451 \quad$ EDUEM. 
452 Revell, L. J. 2009. Size-correction and principal components for interspecific comparative

$453 \quad$ studies. Evolution 63:3258-3268.

454 Revell, L. J. 2012. phytools: an R package for phylogenetic comparative biology (and other 455 things). Methods in Ecology and Evolution 3:217-223.

456 Rodrigues, R. A., Siqueira, M. S., Martins, B. O., Fernandes, C. E., Franco-Belussi, L., Provete, 457 D.B. (2020). Staining of fish Red Blood Cells. protocols.io

$458 \quad$ dx.doi.org/10.17504/protocols.io.bd9yi97w

459 Russ, J.C., Dehoff, R.T. 2000. Practical stereology. $2^{\text {nd }}$ ed. Springer science.

460 Szarski, H. 1970. Changes in the amount of DNA in cell nuclei during vertebrate evolution.

$461 \quad$ Nature 226:651-652.

462 Sekhon, S. S., Beams, H.W. 1969. Fine structure of the developing trout erythrocytes and 463 thrombocytes with special reference to the marginal band and the cytoplasmic $464 \quad$ organelles. American Journal of Anatomy, 125:353-373.

465 Sephton, D. H., MacPhee, W. L., Driedzic, W. R. (1991). Metabolic enzyme activities, oxygen 466 consumption and glucose utilization in sea raven (Hemitripterus americanus) 467 erythrocytes. Journal of experimental biology 159: 407-418.

468 Silkin, Y. A., E. Silkina, V. Chernyaeva, and V. Vasilets. 2019. Study of Dimensional and 469 Morphological Characteristics of Erythrocytes in Some Black Sea Fish of Different 470 Evolution Position and Ecological Specialization. Journal of Ichthyology 59:97-103.

471 Smith, E. M. and T. R. Gregory. 2009. Patterns of genome size diversity in the ray-finned 472 fishes. Hydrobiologia 625:1-25.

473 Tavares-Dias, M., and de Moraes, F. R. 2006. Características hematológicas da Tilapia rendalli 474 Boulenger, 1896 (Osteichthyes: Cichlidae) capturada em "pesque-pague" de Franca, 475 São Paulo, Brasil. Bioscience Journal, 19: 107-114. 
476 Uyeda, J. C., D. S. Caetano, and M. W. Pennell. 2015. Comparative Analysis of Principal

477 Components Can be Misleading. Syst Biol 64:677-689.

478 Wei, T., and Simko, V. "R package "corrplot": Visualization of a Correlation Matrix (Version

479 0.84)". Available from https://github.com/taiyun/corrplot (2017).

480 West, M.J. 2012. Basic stereology for biologists and neuroscientists. Cold Spring harbor

$481 \quad$ laboratory press. New York.

482 Wintrobe, M.M. 1934. Variations in the size and hemoglobin content of erythrocytes in the

483 blood of various vertebrates. Folia haematologica 51:32-49.

484 Witeska, M. 2013. Erythrocytes in teleost fishes: a review. Zoology and Ecology 23:275-281.

485 Yakhnenko, V.M., Klimenkov, I.V., Sudakov, N.P. Belyshenko, A. Y., Glyzina, O. Y.,

486 Mamontov, A. M., Sapozhnikova, Y P. and Sukhanova, L. V. 2016. Morphofunctional

487 peculiarities of erythrocytes in wild and farmed Coregonid fishes from Lake Baikal.

$488 \quad$ Contemporary Problems in Ecology 9: 219-228

489

490

491

492

493

494

495

496

497

498

499

500

501

502

503

504

505

506

507

508

509

510

511

512
Figure Legends

Figure 1. Dated phylogenetic tree with species to which we have phenotypic data used in the analysis, pruned from Rabosky et al. (2018).

Figure 2. Shape and size of Red Blood Cells of the 15 fish species analysed. Staining: MMGW. Scale bars $=10 \mu \mathrm{m}$.

Figure 3. Diagram showing the mean of each stereological variable with the phylogenetic tree of the species sampled. Data on standard deviation for each variable is available at Table $\mathrm{S} 1$.

Figure 4. Phylomorphospace constructed with phylogenetic Principal Components 1 and 2 showing species distribution in the reduced space. Red Blood Cell (RBC) images are all in the same magnification and belong to species occupying the extreme positions along each pPC. The RBC associated positively with pPC1 is from Poecilia, which has a high shape factor, while the RBC associated negatively with pPC1 is from Synbranchus that has high cell area and perimeter, and high nucleus area and perimeter. The RBC associated positively with pPC2 belongs to Pseudoplatystoma, which has high cell perimeter. The RBC image associated negatively with pPC2 is from Astyanax and has a high shape factor, high nucleus:cytoplasm ratio, and high nucleus volume. Colours represent depth in the water column species are usually found, green $=$ Pelagic; black $=$ Benthopelagic; red $=$ Demersal. 
514 Figure 5. Phylogenetic tree showing the results of the tip-level evolution rate estimated

515 using ridge regression for the stereological variables describing the Red Blood Cell

516 shape. Analysis was conducted in RRphylo.

517

518 Data accessibility statement

519

520 All the data and R code used to run the analysis will be deposited in FigShare upon

521 acceptance. 


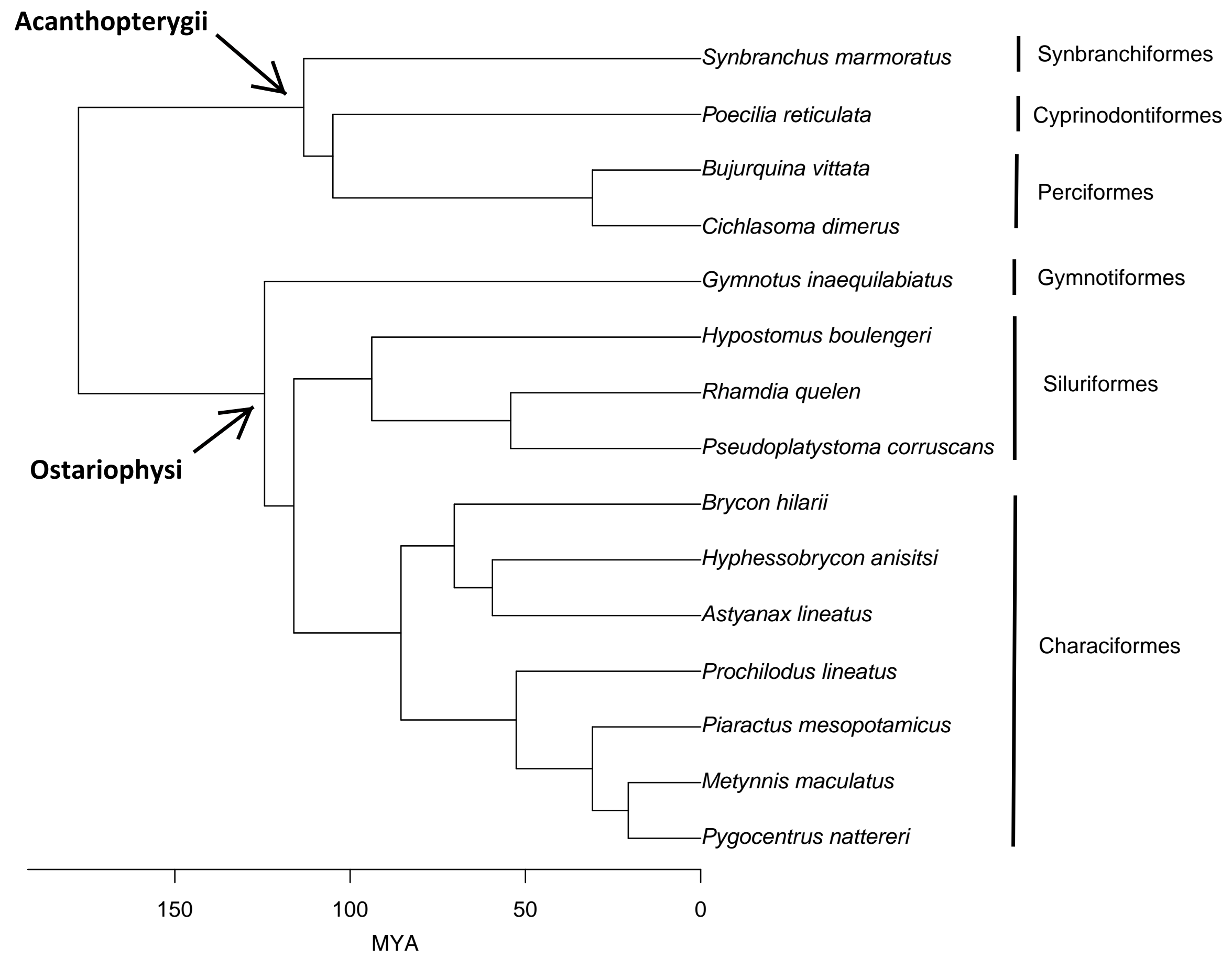




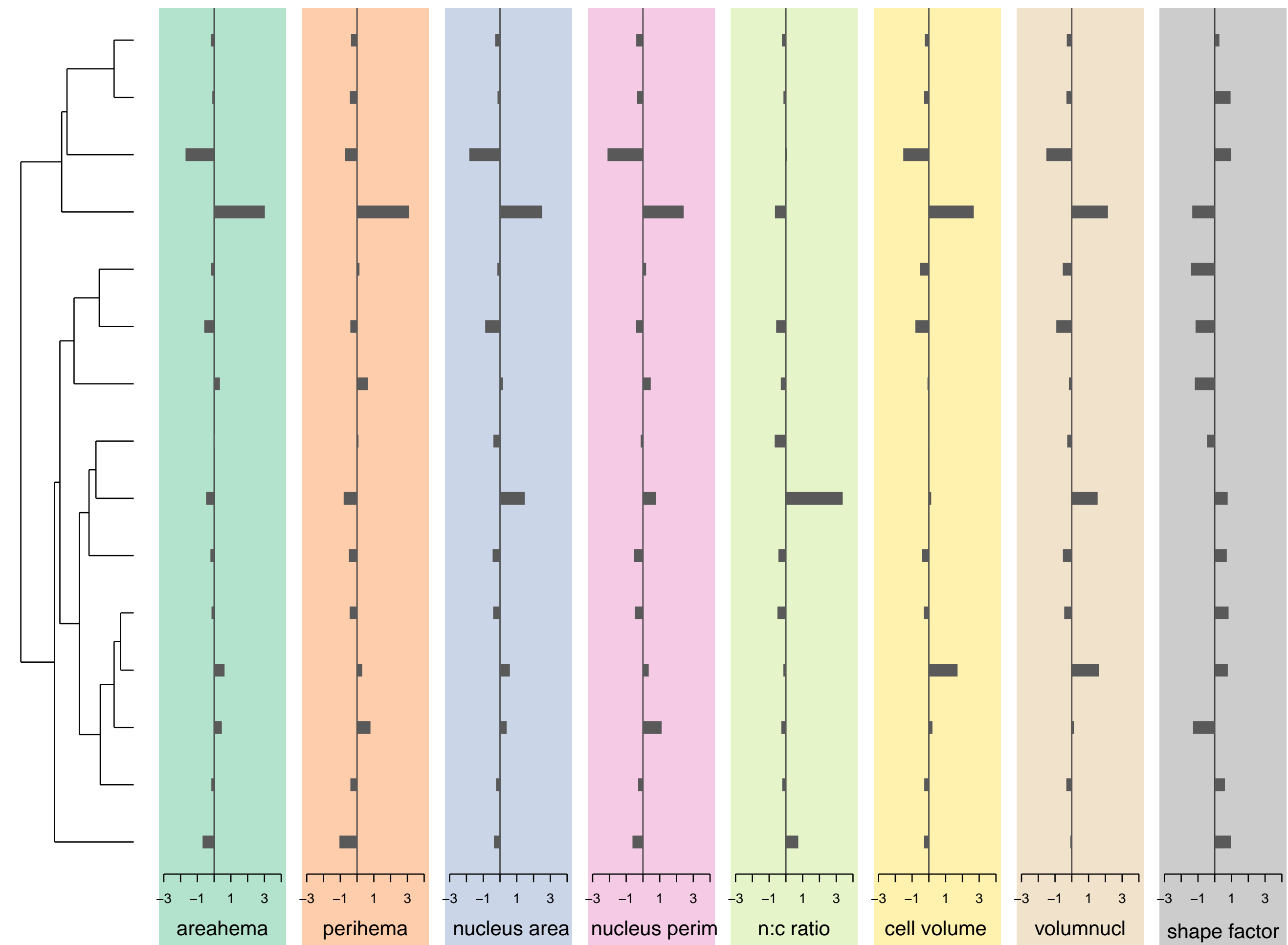

Bujurquina_vittata

Cichlasoma_dimeru

Poecilia_reticulata

Synbranchus marm

Rhamdia_quelen

Pseudoplatystoma

Hypostomus_bouler

Hyphessobrycon_ar

Astyanax_lineatus

Brycon_hilarii

Metynnis_maculatus

Pygocentrus_natter

Piaractus mesopot

Prochilodus lineatu

Gymnotus_inaequilc

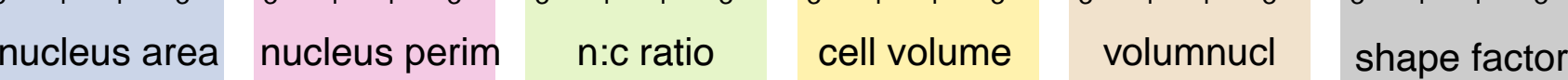









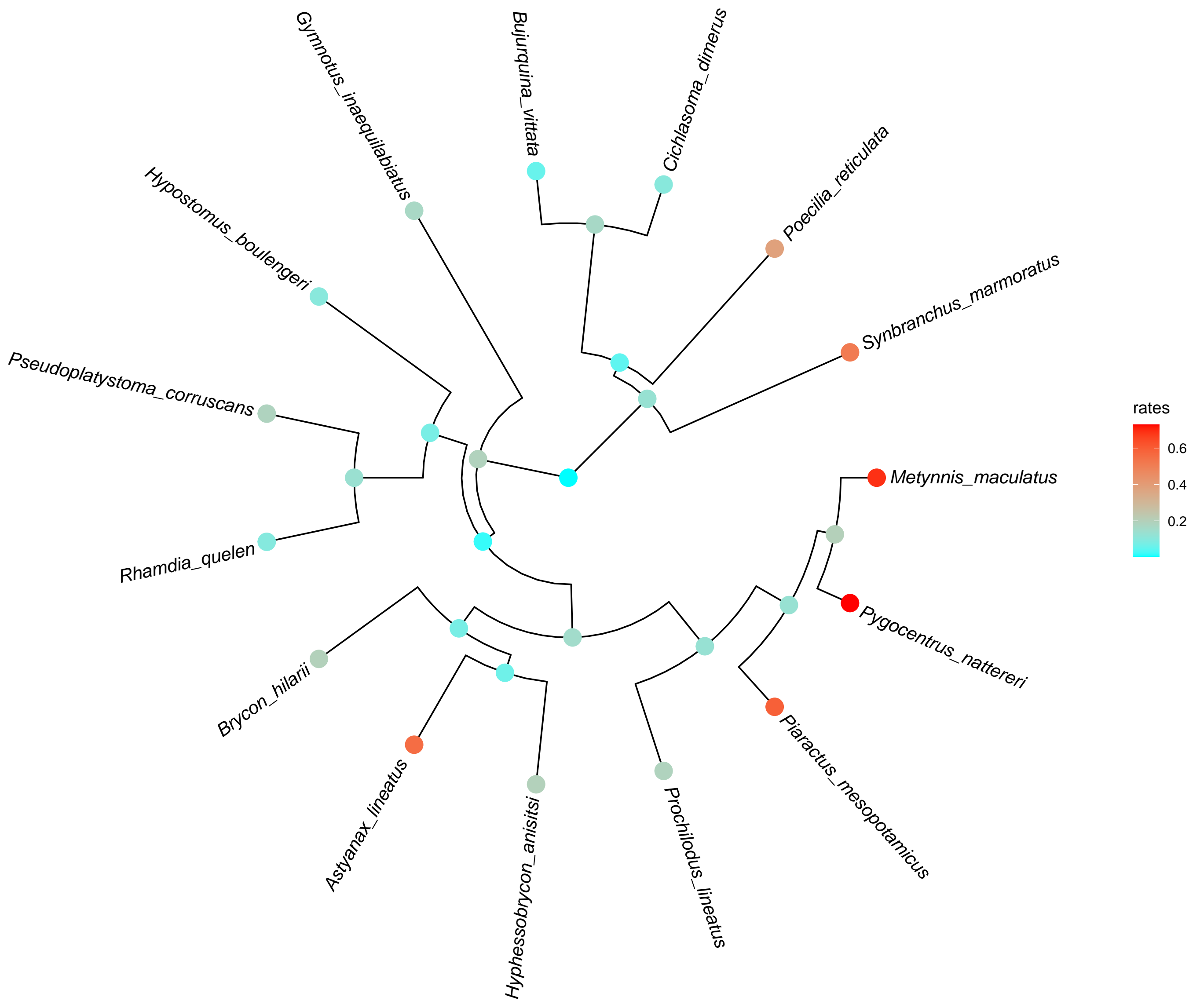

